\title{
GESTANTE Y CONCEBIDO: ENTRE EL DERECHO A LA VIDA Y EL DERECHO A LA MUERTE DIGNA
}

\author{
Sandra Maritza Rovegno Loayza*
}

Recibido: 10.10 .2015

Aprobado: 24.10.2015

\section{RESUMEN}

El presente artículo tiene por finalidad esclarecer la relación jurídica entre madre y concebido en casos en los que la mujer haya fallecido estando embarazada o se encuentre en alguna situación médica de riesgo vital en el Perú. Además, busca determinar los derechos de cada uno de estos sujetos en tales circunstancias. Para ello, se han empleado criterios legales y médicos; además de jurisprudencia internacional y principios de Bioética. Por otro lado, también se hace énfasis en que es necesario emplear criterios multidisciplinarios para poder lograr el mejor resultado en dichos casos.

\section{ABSTRACT}

This article aims to clarify the legal relationship between mother and unborn child in cases where the woman has died while pregnant or is in any life-threatening medical situation in Peru. It also seeks to determine the rights of each of these subjects in such circumstances. In order to do this, legal and medical criteria have been used; as well as international jurisprudence and Bioethics principles. On the other hand, emphasis on the need to use multidisciplinary approaches to achieve the best result in such cases is also made.

\section{PALABRAS CLAVE}

Concebido. Derecho a la vida. Autodeterminación. Derecho a la muerte digna. Bioética. Muerte.

\section{KEY WORDS}

Unborn child. Right to life. Self-determination. Right to a Death with Dignity. Bioethics. Death.

\section{A MODO DE INTRODUCCIÓN}

La relación entre una madre gestante y el concebido que lleva en el vientre, cuando existen derechos que pueden contraponerse, es un tema que suele generar polémica en ámbitos religiosos, médicos, sociales, políticos, entre otros. Es evidente que surjan divergencias en torno a este asunto dado que puede existir una yuxtaposición entre los derechos de ambos sujetos. Las disputas alrededor de esta cuestión varían desde aspectos muy simples hasta otros bastante complejos. Los debates que surgen acerca del aborto, del alquiler de vientres, las distintas posturas acerca del momento preciso en el que se produce la concepción, los múltiples criterios científicos propuestos para determinar el inicio de la vida, entre otros, son frecuentes alrededor del mundo.

Los casos extremos como, por ejemplo, los que implican la vida de alguno de los dos, forjan una serie de aristas que dificultan determinar el tratamiento legal que se le debería

* Asistente de Investigación en el Instituto de Investigación Científica de la Universidad de Lima (IDIC). Estudiante del último año de Derecho en la Universidad de Lima. 
dar a cada individuo. Ponderar entre el derecho a decidir de la madre -o, en su defecto, el de sus familiares sobre el cuerpo de esta si es que ella se encuentra incapacitada de hacerlo- y la vida del concebido; decidir entre el derecho a la libre determinación del cuerpo humano y la protección del nasciturus ${ }^{1}$; lograr una determinación jurídica uniforme acerca del momento preciso en el que se debe considerar al feto un ser humano, y muchos supuestos más, son algunas de las situaciones relacionadas con el Derecho que corresponde a nuestra sociedad afrontar.

Una de las figuras menos comunes, pero, también, más controvertidas que se han presentado al respecto es el caso en el cual la mujer fallece o queda en estado de coma durante el periodo de gestación y el concebido se mantiene con vida. A pesar de ser un suceso poco común, ha causado mucho revuelo legal y mediático cuando se ha presentado. Existe jurisprudencia al respecto, con distintas connotaciones, en países como Estados Unidos e Irlanda², pero, ¿cuál sería el tratamiento jurídico de este tipo de situaciones en el Perú? Usualmente, la disputa ha surgido respecto de tres aspectos principales, que serán materia de desarrollo en el presente artículo: el inicio y el fin de la vida, los derechos de la madre y los derechos del concebido.

Existen múltiples preceptos a tener en cuenta respecto de este tema. En primer lugar, es necesario resaltar que el concebido es un sujeto de derecho favorecido por nuestra legislación: es titular de especial protección. Teniendo en cuenta ello, podríamos sentar la idea de que, en el Perú, el concebido será resguardado en todo lo que sea pertinente y en la medida de lo posible. Por otro lado, también es importante plantear el concepto, que muchos apoyan, acerca de que el organismo de la gestante deberá tener un especial cuidado en tanto depende de él la vida del ser humano por nacer. En segundo lugar, es esencial tener en consideración el estatus jurídico del cuerpo de la madre, ya que, si bien se trataría de un cadáver o de un ser humano sobreviviendo solo gracias al soporte vital médico, ¿sería este un simple cadáver o el hecho de tener un ser vivo dentro de ella le otorgaría especial tratamiento?

\section{SOBRE EL INICIO Y EL FIN DE LA VIDA HUMANA}

El Código Civil peruano se pronuncia acerca del inicio de la vida, de manera concisa, en el artículo 1 del Libro I. El texto refiere lo siguiente:
"Artículo 1.- La persona humana es sujeto de dere- cho desde su nacimiento.
La vida humana comienza con la concepción. El con- cebido es sujeto de dere- cho para todo cuanto le favorece. La atribución de derechos patrimoniales está condicionada a que nazca vivo."

Es claro, entonces, que, para nuestro ordenamiento jurídico, la concepción es el momento en el cual el ser humano emprende el camino de la existencia. Por tanto, desprendemos de esta afirmación que el concebido es tratado como un ser humano, aunque este, evidentemente, aún no haya sido alumbrado. ${ }^{3}$

Resulta esencial, para efectos de comprender plenamente dicha reflexión, lo que implica la concepción. Sobre ello, el Tribunal Constitucional del Perú se ha pronunciado de la siguiente manera:

“(...) Desde el punto de
vista de la ciencia médi-
ca existen diversas teorías
que pretenden identificar
el momento en el que la
vida humana empieza. Hay
quienes consideran que la

\footnotetext{
1 “El «nasciturus» es el concebido aún no nacido («nondum natus»), es el ser humano en el periodo de su vida que va desde el momento de la concepción hasta el momento del nacimiento, y se desarrolla en las diferenciadas etapas de embrión y de feto." (Calvo Mejide, 2004, p. 291)

2 Para más detalles, revisar: http://www.irishtimes.com/news/crime-and-law/courts/high-court/court-clears-wayfor-clinically-dead-pregnant-woman-to-be-taken-off-life-support-1.2048616 (Consultada el 02.07.2015)

3 El Código de los Niños y Adolescentes (Perú) hace referencia a esta misma idea en su artículo I, en el cual, establece lo siguiente:

"Artículo I.- Definición.- Se considera niño a todo ser humano desde su concepción hasta cumplir los doce años de edad y adolescente desde los doce hasta cumplir los dieciocho años de edad. El Estado protege al concebido para todo lo que le favorece. Si existiera duda acerca de la edad de una persona, se le considerará niño o adolescente mientras no se pruebe lo contrario."

Nuevamente, el Estado peruano incluye al concebido en la categoría de ser humano, a pesar de aún no haber nacido.
} 
vida humana surge desde el instante en que se inicia la actividad cerebral (aproximadamente la sexta semana contada desde la fecundación), pues resulta lógico que si la persona llega a su fin con el estado irreversible de las funciones cerebrales, de la misma manera la actividad cerebral daría inicio a la vida. Sin embargo, las más importantes considerando el número de seguidores, y que justamente han sido ampliamente debatidas a partir del caso en cuestión, se encuentran en la llamada Teoría de la Fecundación, basada principalmente en la existencia, ya en esta instancia, de una nueva individualidad genética; y la Teoría de la Anidación, fundamentada en la viabilidad del embrión y la certeza del embarazo. (...)"'4

En términos generales, la teoría a la que se ha hecho más referencia en nuestro país -y que tiene respaldo constitucional- es la de la Fecundación, en tanto que nuestro ordenamiento protege al concebido y la manera más amplia de hacerlo es asumir que la vida humana existe desde que se genera un ser, genéticamente, diferente de los progenitores.

Acerca de esto, algunos autores como Mónica López Barahona y José Carlos Abellán $(2009)^{5}$, se apoyan en criterios científicos objetivos para sustentar su postura, la cual afir- ma que el cigoto humano, por ser poseedor de un genoma de secuencias Alu ${ }^{6}$-y al tratarse, además, de una nuevo ente- es un tipo de vida humana plenamente reconocible.

Una vez establecido lo que, en el Perú, el Estado considera el inicio de la vida humana, surge la interrogante acerca de cuándo es que termina la misma. Sobre ello, el Código Civil también se ha pronunciado de manera bastante breve en el Artículo $61^{\circ}$, cuyo texto señala: "La muerte pone fin a la persona." Acerca del concepto de la muerte existen múltiples posturas que se han desarrollado a lo largo de los años e, incluso hoy, resulta complejo determinar, exactamente, cuál es el momento en el que la vida de una persona llega a su fin.

Sobre esta cuestión médica y jurídica, Juan Morales Godo (1997) ha realizado una apreciación bastante certera en su libro "Hacia una Concepción Jurídica Unitaria de la Muerte", la cual refiere:

“(...) dentro del proceso
biológico de la muerte, tan-
to la medicina como el De-
recho, habían determinado
el momento de la muerte
cuando cesaba el funciona-
miento de los tres sistemas
superiores del ser humano
(con esto hace referencia al
sistema cardiovascular, al
respiratorio y al nervioso),
pero, como también hemos
precisado, el cese no se

produce en forma simultánea, sino uno es consecuencia del otro. Descartados los sistemas respiratorio y el cardiovascular, como situaciones biológicas absolutamente irreversibles, debido al avance de las técnicas de reanimación, se ha recurrido al concepto de la muerte cerebral (...)"7. (Morales, 1997, p.30)

Como muerte cerebral, debemos comprender, entonces, el cese del funcionamiento del cerebro de una forma irreversible, lo cual, evidentemente, establece un criterio para comprender lo que es la muerte del ser humano, pero gene$\mathrm{ra}$, a su vez, numerosos posibles eventos en los que puede existir controversia. Un ejemplo de ello, es la situación de las personas cuya actividad respiratoria y cardiovascular se encuentran condicionadas a equipos médicos $y$, a su vez, no cuenten con esperanza de recobrar sus funciones cerebrales habituales.

\section{CRITERIOS mÉdico-JuRÍdICOS}

Por distintos motivos -tanto religiosos como morales y científicos- el ser humano, a lo largo de su historia y en múltiples civilizaciones, ha optado por retirar al concebido del cuerpo de la madre una vez que esta ha perdido sus facultades vitales. La principal valoración que se ha empleado para sustentar esto ha sido el interés por darle una oportunidad de vida al niño por nacer. Esto úl-

Exp. N. ${ }^{\circ}$ 02005-2009-PA/TC - Lima, 16 de octubre de 2009.

López, M. y Abellán, JC. (2009). Los códigos de la vida, p. 9.

Para más detalle, revisar: http://www.unav.es/ocw/genetica/tema-1-3.html (Consultada el 01.10.15).

Morales Godo, J. (1997). Hacia una Concepción Jurídica Unitaria de la Muerte. Lima, PUCP, p. 30. 
timo nos remite, nuevamente, al precepto que, en la actualidad, lo que se busca, sustancialmente, es darle seguridad al concebido y favorecerle en todo aquello que sea posible.

No obstante, no podemos ceñirnos únicamente a criterios jurídicos o morales para determinar si existe posibilidad de salvar esa nueva vida; es por ello que los médicos, en términos generales, han establecido determinadas características objetivas que debe tener un caso para poder realizar un procedimiento de separación exitoso, también llamado operación cesárea postmortem con supervivencia fetal. Sobre ello, Arturo Juárez Azpilcueta y otros autores, señalan en su artículo "Operación cesárea postmortem con supervivencia fetal. Informe de un caso" (1999), como principales factores esenciales, los siguientes:

“1. Edad gestacional. El límite inferior para lograr posibilidades de éxito es la semana 28 de gestación.

2. Estado del feto. Se recomienda realizar la operación independientemente de tener la certeza de viabilidad fetal. En caso de que el feto esté vivo y en condiciones satisfactorias, las posibilidades de éxito son mayores.

3. Tiempo que transcurre entre la muerte materna $y$ el nacimiento del feto.
El tiempo que transcurre entre la muerte materna y el nacimiento del feto es otro factor que determina el éxito de operación. Se ha informado que el tiempo ideal son los primeros 20 minutos, aunque existen casos de supervivencia fetal posterior a los $22 \mathrm{mi}$ nutos. Sólo se ha registrado en la literatura un caso de supervivencia fetal después de 45 minutos de ocurrido el deceso de la madre.

4. Motivo de la muerte materna. La diferencia entre la muerte de una madre con un padecimiento crónico y aquella que ocurre en forma repentina e inesperada, radica en la condición general de hipoxia que ocasiona la primera situación; en este caso, para el feto el pronóstico es más pobre que cuando suceden los acontecimientos del segundo caso.

5. Adecuada y rápida reanimación del recién nacido. Es una medida lógica entender que, si la persona a la que le toque la reanimación del recién nacido conoce el suceso previo y cuenta con los recursos disponibles para la rápida reanimación del recién nacido, el pronóstico para la supervivencia fetal se verá favorecido." (Juárez et al., 1999. p. 134).

Resulta sustancial, entonces, comprender que, si bien la le- gislación establece que el concebido es un sujeto de derecho especial, en tanto goza de especial cuidado y atención, médicamente, este trato favorecido estará delimitado por una serie de criterios imparciales, y que dependerán del caso en particular los límites y limitaciones de lo que se pueda realizar por el mismo.

Como sucede en muchas ocasiones, el Derecho, al encontrarse íntimamente vinculado con el bienestar social, debe recurrir a criterios multidisciplinarios para poder idear una solución justa y objetiva a este tipo de situaciones. Esto acaece, sobre todo, hoy en día, cuando los avances legales deben apoyarse, muchas veces, en los que la ciencia realiza a pasos acelerados.

\section{Derechos de la Madre}

Al referirse a los derechos relativos a la madre, es importante, en primer lugar, establecer que la gestante, por sobre todas las cosas, es un individuo. En otras palabras, es esencial comprender que la mujer, en todo extremo, es titular de los derechos inherentes que le corresponden como ser humano. No obstante ello, también resulta primordial poner énfasis en la premisa de que sus derechos serán efectivos, para nuestra legislación, en tanto no perjudiquen directa y arbitrariamente a los del concebido.

8 Juárez, A.; Carrillo, C.; Romero,S.; Motta, E. (1999) "Operación cesárea postmortem con supervivencia fetal. Informe de un caso”. En: Revista Médica del Hospital General de México S.S.. Vol. 62, Núm. 2. Abril-Junio, p. 134.

9 Sobre ello, en 1970, el Colegio Médico del Perú se pronunció de la siguiente manera: “Cada caso de aborto terapéutico que se plantee en el ejercicio de la profesión debe resolverse a criterio de una junta médica de no menos de tres especialistas en el problema que se trate. Si llega a concluirse en la junta médica que es procedente el aborto terapéutico, éste debe efectuarse en un hospital o clínica oficialmente reconocida" - Resolución CMP/CN-20, del 4 de noviembre de 1970. 
En este artículo nos enfocaremos en sintetizar los derechos más importantes que conciernen a la mujer gestante y cómo se desarrollarán estos en determinados casos en los cuales la mujer se vea inmersa en circunstancias que la conduzcan a entrar en estado de coma o en los cuales su vida pudiese encontrarse en riesgo. En eventos que involucren peligro, podemos deducir que existen grandes tipos de derechos que desempeñarán roles significativos: por un lado, los vinculados a la vida y salud $y$, por otro, los relacionados con la libertad y la voluntad.

Respecto del derecho a la vida, nuestro ordenamiento contempla que, en el caso en el que la gestante se encuentre en riesgo de perderla, dada su condición, será legal la alternativa del llamado "aborto terapéutico". Sobre esto, el Código Penal señala:

\begin{abstract}
"Aborto terapeútico Artículo 119. - No es punible el aborto practicado por un médico con el consentimiento de la mujer embarazada o de su representante legal, si lo tuviere, cuando es el único medio para salvar la vida de la gestante o para evitar en su salud un mal grave y permanente."
\end{abstract}

Debemos considerar, también, que existen cuestiones en las cuales algunos sectores de la doctrina podrían considerar a la mujer como el vehículo para que el niño por nacer se mantenga con vida - por ejemplo, cuando la madre se encuentra en estado de coma o cuando ha padecido muerte cerebral-. No obstante, en tales circunstancias, la jurisprudencia internacional parece presentar una tendencia a anteponer los derechos de la mujer a tener una existencia digna y de la familia a decidir respecto del destino de esta antes que plantear los derechos del concebido como eje principal para decidir respecto de la madre. Sin embargo, debemos resaltar que las consideraciones médicas que mencionamos en párrafos anteriores también se configuran como un importante punto a considerar.

Por otro lado, cuando nos referimos a la voluntad de la madre, en el Perú, legalmente, esta estará condicionada a no hacerle daño al concebido. En otras palabras, en nuestro país, el derecho a la libertad de decisión de la madre se verá limitado por el derecho a la vida de su hijo por nacer; con la salvedad antes mencionada. En situaciones en las que la madre no pueda expresar su voluntad, se deberá tener en cuenta la conformidad o disconformidad de los familiares corres- pondientes para cualquier consideración médica que pudiese llegar a ser necesaria, tal como sucede en otros sistemas jurídicos, sobre todo, en el anglosajón. ${ }^{10}$

Así mismo, es importante considerar lo que indica la Ley General de Salud del Perú (Ley $N^{\circ}$ 26842):

"Artículo 108.- La muerte pone fin a la persona. Se considera ausencia de vida al cese definitivo de la actividad cerebral, independientemente de que algunos de sus órganos o tejidos mantengan actividad biológica y puedan ser usados con fines de transplante, injerto o cultivo. El diagnóstico fundado de cese definitivo de la actividad cerebral verifica la muerte. Cuando no es posible establecer tal diagnóstico, la constatación de paro cardiorespiratorio irreversible confirma la muerte. Ninguno de estos criterios que demuestra por diagnóstico o corroboran por constatación la muerte del individuo, podrán figurar como causas de la misma en los documentos que la certifiquen."

Por lo que debemos inferir que, en nuestro país, en términos generales, la muerte cerebral indica el fallecimiento.

10 En el caso "Munoz v. John Peter Smith Hospital” ocurrido en Estados Unidos entre los años 2013 y 2014 , un juez del Estado de Texas determinó que la cláusula del “Texas Advance Directives Act of 1999" que indicaba: "a person may not withdraw or withhold life-sustaining treatment ... from a pregnant patient." no era aplicable al caso de la señora Muñoz, quien tras sufrir una embolia pulmonar, padeció muerte cerebral estando embarazada. Por lo tanto, la pretensión del Hospital de mantener a la paciente viva artificialmente hasta que el feto pudiese nacer fue desestimada, ya que se consideró que, al estar cerebralmente muerta y supeditada a la ayuda vital de máquinas, no se le podía observar, en realidad, como un ser humano aún vivo, por lo que la decisión de la familia de desconectarla -guiados por la voluntad estipulada por la víctima previa a los sucesos que la llevaron a la muerte- con el objeto de darle una muerte digna, debía primar. 
Desde el punto de vista de la bioética, aún no existen criterios uniformes cuando nos referimos al tema mencionado en el párrafo anterior. Por lo que, una misma situación podrá resultar profundamente distinta para ciertos sectores: la gestante diagnosticada con muerte cerebral podrá ser considerada un cadáver funcionando como una "incubadora" para el niño por nacer o podrá ser tratada como un ser humano en una etapa terminal de la vida, que se encuentra aguardando a ser desconectada.

Sobre ello, es interesante un caso mencionado por Elio Sgreccia, en su "Manual de Bioética I - Fundamentos y ética biomédica":

"En Alemania el debate se reabrió en octubre de 1992 cuando, después de un accidente de tráfico, una mujer joven entró en coma y, tras las comprobaciones previstas, fue declarada cerebralmente muerta. Con el consentimiento de sus padres, los médicos pretendían proceder a la extracción de órganos, pero se dieron cuenta de que la mujer estaba embarazada. Este hecho influyó sobre la decisión de los médicos disponibles para permitir la prosecución del embarazo. La discusión sobre la muerte cerebral se encendió en Alemania y fueron muchos los que se preguntaron cómo podía ser posible para un <<cadáver >> llevar adelante un embarazo."11 (Sgreccia, 2009, p.823)

Asimismo, existe otro derecho que debe tenerse en consideración cuando nos encontramos ante estos episodios: el de la muerte digna u ortotanasia. Si bien este término suele ser vinculado con el de la "eutanasia", en realidad, abarca mucho más de lo que, usualmente, se imagina. La ortotanasia trata del respeto a la existencia humana y al fin de esta. Abarca, de esta manera, muchos conceptos médicos y jurídicos, como, por ejemplo: el suicidio asistido en sus diversas modalidades, el rechazo a continuar conectado a aparatos de soporte vital, entre otros. En pocas palabras, se conoce como:

“(...) el arte de morir bien, humanamente y correctamente, sin ser victimado por la mistanasia, por un lado, o por la distanasia, por otro, y sin abreviar la vida, o sea, recorrer a la eutanasia. Tiene como un gran desafío el rescate de la dignidad del ser humano en su proceso final, donde hay un compromiso con la promoción del bienestar de la persona en su fase terminal."12 (Amorin et al., 2009).

Aunque este concepto no es tratado a cabalidad en nuestra legislación -con la salvedad de determinadas menciones como la que se realiza en el Código Penal respecto al suicidio asistido $^{13}$ - podemos interpretar que, el considerar la muerte cerebral como el fin de la vida humana, de cierta manera, es un gran paso en dirección del camino hacia el respeto que merece el ser humano no solo durante su vida, sino también al final de esta.

Resulta estremecedor, al revisar casuística médica en la cual la gestante con muerte cerebral se ha mantenido conectada, encontrar que, en la gran mayoría de casos, el cuerpo de la mujer ha sufrido complicaciones médicas. Algunas de las consecuencias físicas padecidas han sido: diabetes insípida, infecciones, hipotensión inmanejable, fiebre, anemia, hipertensión renal, inestabilidad hemodinámica, entre otras. ${ }^{14}$

Esto demuestra que, objetivamente, para intentar salvar artificialmente al concebido, se tendrá que exponer al cuerpo en funcionamiento artificial

11 Sgreccia, E. (2009). Manual de Bioética I - Fundamentos y ética biomédica. Madrid. Biblioteca de autores cristianos, p.823.

12 Amorim Biondo, C.; Paes da Silva, MJ; Dal Secco, LM. (2009). “Distanasia, eutanasia y ortotanasia: percepciones de los enfermeros de unidades de terapias intensiva e implicaciones en la asistencia" En: Rev Latino-am Enfermagem setembro-outubro; 17(5) (Online). Disponible en internet: http://www.scielo.br/pdf/rlae/v17n5/es_03.pdf (Consultado el 29.09.15).

13 El Código Penal Peruano hace referencia, de cierta manera, a este concepto con el siguiente tenor: “Instigación o ayuda al suicidio - Artículo 113. - El que instiga a otro al suicidio o lo ayuda a cometerlo, será reprimido, si el suicidio se ha consumado o intentado, con pena privativa de libertad no menor de uno ni mayor de cuatro años. La pena será no menor de dos ni mayor de cinco años, si el agente actuó por un móvil egoísta."

14 Mejía R. et al. (2008) "Muerte cerebral en una embarazada y sobrevida del feto". ISSN 0025-7680 En: MEDICINA, Buenos Aires: p. 449 
de la madre a enfermedades, lo cual nos transmite una inquietud importante: ¿será legal y ético este tratamiento? Es esencial resaltar que cualquier práctica médica debe siempre ir acorde a lo que las buenas costumbres permiten y debe tener por fin el bienestar humano, lo cual no necesariamente sucedería siempre en casos como estos.

Esta suerte de "vida extra" que estaría experimentando la mujer con muerte cerebral no podría ser, de ninguna manera, natural. A diferencia de tratamientos médicos en los cuales se emplea la ciencia para prolongar la subsistencia de la persona natural, en este caso, se estaría empleando la existencia del individuo con fines externos a este. Deberían, entonces, los médicos, además de remitirse a criterios técnicos, a la voluntad de la paciente, si esta la expresó anticipadamente o, en todo caso, a la de sus familiares.

En casos en los que la madre fallece de manera súbita e irreversible, resulta bastante más sencillo determinar cuál será el destino del concebido. Este deberá ser extraído del cuerpo materno si así lo permite su grado de crecimiento para, de esta manera, otorgarle una oportunidad de vida. El cuerpo de la gestante fallecida recibiría, como consecuencia, el trato digno del que todos los restos humanos son merecedores.
Finalmente, debemos hacer mención al derecho a la libre disposición del propio cuerpo, del cual es titular la mujer. Acerca de ello, es trascendente resaltar que este se encuentra delimitado, en su propia esencia y a fin de no perjudicar a otros sujetos, por conceptos como la satisfacción de un estado de necesidad de orden médico, los fines terapéuticos, el bien común, entre otros.

\section{Derechos del concebido}

Si bien, históricamente, no siempre se consideró al concebido titular de derechos, este, desde la época de los romanos, ya contaba con especial protección: "Los profesores J. Arias Ramos y J.A. Arias Bonet opinan que el derecho romano no reconoció al concebido como sujeto de derecho, sino que solo protegió sus intereses y su futura persona. Los principios doctrinales no daban personalidad jurídica al nasciturus, sino protección."15 (Cruz Ponce, 1990, p.67).

\section{Partiendo del postulado} kantiano que sostiene que la persona, en sí misma, es un fin; podemos inferir que el concebido, en tanto para el sistema jurídico peruano es una forma de vida humana, es titular de derechos inherentes, los cuales no son parte de la esfera jurídica de sus progenitores, sino de una propia. En palabras de Fernández Sessarego (1990) “(...) El conce- bido es un ser humano que, aunque incapaz de entender $y$ de querer- y hasta un cierto momento de sentir- es un fin en sí mismo y no puede ser reducido a la calidad de medio o instrumento para lograr otros fines.". ${ }^{16}$

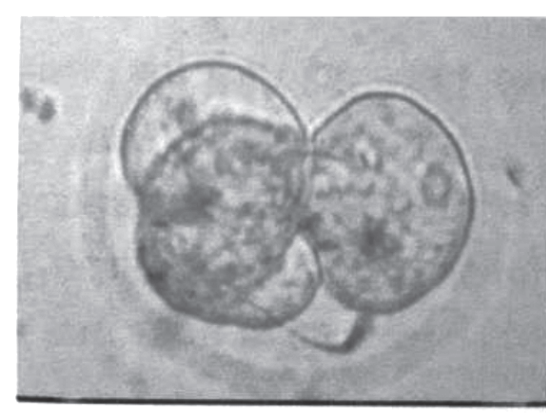

Cigoto en segmentación (cuatro células) a los tres días postfecundación.

(Figura adaptada de "Enciclopedia Lúmina Siglo XXI" [Candelaria, et al. 2004] p. 203)

El derecho más importante al que debemos referirnos cuando tratamos la esfera legal del concebido, en este tipo de situaciones, es el de la vida. Como mencionamos anteriormente, el inicio de la vida, para nuestra legislación, se produce con la concepción; por ello, debemos entender que, en el Perú, en tanto exista individualidad genética, este sujeto de Derecho será protegido en todos los extremos posibles.

Para profundizar la posición del Estado Peruano acerca de este tema -la cual ha sido mencionada en párrafos anteriores-, nos remitimos a lo expuesto por Rosario de la Fuente-Hontañón (2011):

\footnotetext{
15 Cruz Ponce, L. (1990) “El nasciturus”. En: Boletín Mexicano de Derecho Comparado, México, nueva serie, año XXIII, núm. 67, enero-abril, 1990, p.38.

16 Fernández Sesarego, C. (1990). Nuevas Tendencias en el Derecho de las Personas. Lima: Universidad de Lima, p. 67.
} 
"Se denomina embrión al ser humano desde el momento de la fecundación, esto es, desde el momento de la unión del óvulo y el espermatozoide hasta los primeros meses de vida. Se trata de un individuo de la especie humana distinto y dinámicamente autónomo respecto de la madre cuyo desarrollo es un proceso continuo ordenado por tres principios: la coordinación - de acuerdo a la información contenida en el genoma-, la continuidad y la gradualidad. En este punto existe un amplio acuerdo en la doctrina científica, aunque algunos autores apoyándose en los datos que nos dan las ciencias biológicas niegan la condición individual del cigoto."17 (De la Fuente-Hontañón, 2011).

En otras palabras, si bien el cigoto no es físicamente independiente, en la medida de que no puede valerse por sí mismo para subsistir, sí lo será respecto de su identidad biológica. Es decir, se trata de un "ser humano genéticamente individualizado" (Fernández Sessarego, 1988, p.52) ${ }^{18}$.

En el siguiente gráfico, que hemos elaborado, podemos observar el momento en el cual, de acuer- do con esta postura, luego de la unión de los gametos, se empieza a desarrollar un nuevo individuo:

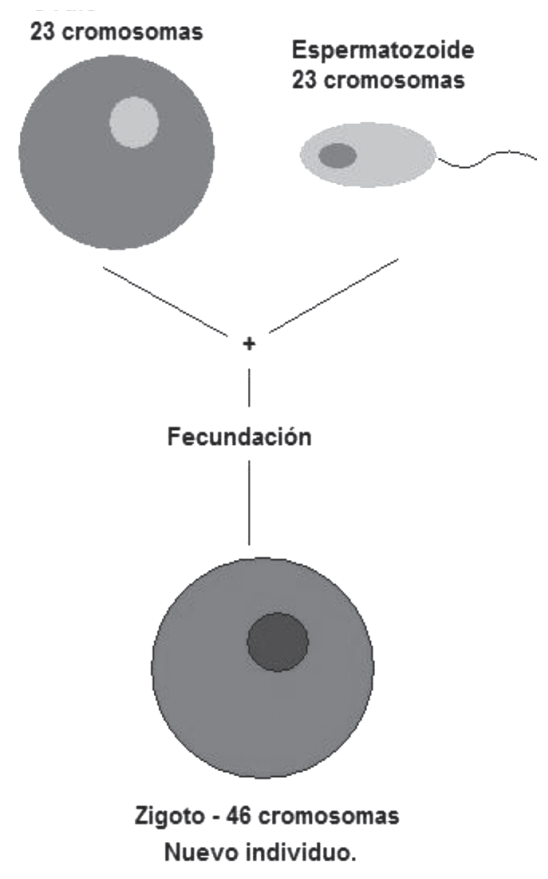

Nuestra legislación no es ajena, entonces, a la postura de una parte de la sociedad científica que considera que el proceso de la evolución del embrión da inicio desde antes de la implantación del mismo en el útero materno. ${ }^{19}$

No se tendrá en cuenta, por consiguiente, en la legislación peruana, que el inicio de la vida se produce con la aparición de la actividad cerebral en el concebido alrededor de los cuatro meses de embarazo. Esto sobre la base que, desde el momento de la fecundación, existe viabilidad para la vida humana y, por lo tanto, protección para la misma. La postura del Estado resulta discutible, para ciertos sectores, dado que se considera que, si la existencia humana finaliza con el cese de la actividad cerebral; debería iniciar cuando esta aparece y no ampararse en potencialidades para generar derechos y obligaciones. ${ }^{20}$

Por el contrario, en el Perú, el asunto reside en que, legalmente, el concebido como humano- existe a partir de la fecundación, cuando, evidentemente, se encuentra en el sistema de la madre. Será, entonces, cuando se le considere titular de determinados derechos, que se terminan de afirmar al momento del nacimiento -o finalizan con el término de la existencia del no nacido-. En este último caso, jurídicamente, se considerará que el concebido nunca existió. ${ }^{21}$

Una vez establecido esto, surge una nueva interrogante. ¿En qué medida se considera al concebido un sujeto de derecho que depende de su madre para poder hacer efectivo su derecho a la vida? En principio, debemos responder que, en tanto el concebido no puede valerse por sí mismo, estará

17 De la Fuente-Hontañón, Rosario (2011). "La protección de la vida y la dignidad de la persona humana en el derecho peruano.” En: Repositorio institucional PIRHUA (Online). Universidad de Piura, 2011. Disponible en internet: http: // pirhua.udep.edu.pe/bitstream/handle/123456789/1632/Proteccion_de_la_vida_y_dignidad_de_la_persona_ humana.pdf?sequence=1 (Consultado el 29.09.15).

18 Fernández Sessarego, C. (1988) "Tratamiento jurídico del concebido", en Libro Homenaje a Mario Alzamora Valdez. Lima: Cultural Cuzco, p. 52.

19 Busquets et al. (2009) "Consideraciones sobre el embrión humano". En: Bioética y debat. Volumen 15, Número 57, Monográfico 2009. (Online). Disponible en internet: http://www.ibbioetica.org/es/img/home/BD_57_esp_F (Consultado el 10.07.15).

20 González, L. (2006) “De la bioética-al bioderecho: libertad, vida y muerte”. Universidad Pontifica Comillas, p. 480.

21 Spota, AG. (1949). Tratado de Derecho Civil. Tomo I, Volumen 3". Buenos Aires: Editorial Depalma, p. 49. 
condicionado a que la madre le proporcione una existencia protegida de cualquier daño o amenaza.

Es importante comprender que, cuando en párrafos anteriores mencionábamos determinados criterios médicos para medir las posibilidades que tendría el concebido para sobrevivir si algo le ocurriese a su madre, incluso bajo esas condiciones, él continuaba dependiendo de que el cuerpo de la madre continuase funcionando -inclusive artificialmente- para brindarle un ambiente propicio para existir. Sobre ello, podemos referir:

“(...) la futura madre se ha ubicado a sí misma en una clase especial de personas que están trayendo a otra persona a la existencia $y$, sobre quien, la vida de esa otra persona es totalmente dependiente. También, de forma única, el niño no-nacido viable se encuentra, literalmente, cautivo dentro del cuerpo de la madre (...)"22 (Friedman, 1994, p.164)

¿Es, entonces, un cadáver el ambiente propicio para que la vida humana pueda desarrollarse?

Si nos ceñimos a lo planteado por parte de la doctrina, la mujer con muerte cerebral no será sino un cuerpo despojado, naturalmente, de vida cuyos demás órganos continúan funcionando únicamente gracias al soporte vital que los médicos puedan proporcionarle. En teoría, entonces, cuando la madre fenece, el concebido que no se encuentre en condiciones de sobrevivir como un niño nacido a ese punto, debería también decaer de manera natural con su progenitora.

Si bien es cierto que, muchas veces, podría ser médicamente posible mantener el funcionamiento del cuerpo de la mujer perecida hasta que el no nacido alcance determinado grado de crecimiento, resulta de suma importancia considerar que, para muchas personas, podría implicar un proceder poco ético el emplear un cuerpo humano fallecido como una suerte de máquina para lograr obtener a otra persona. Este punto de vista es ampliamente reconocido en el mundo occidental hoy en día.

\begin{tabular}{|c|c|c|c|}
\hline & $\begin{array}{l}\text { Muerte } \\
\text { cerebral }\end{array}$ & Coma & $\begin{array}{c}\text { Estado } \\
\text { Vegetativo } \\
\text { Persistente } \\
\text { (EVP) }\end{array}$ \\
\hline Conciencia & No hay & No hay & No hay \\
\hline Sueño-vigilia & No hay & No hay & $\begin{array}{l}\text { Existe } \\
\text { alternancia }\end{array}$ \\
\hline $\begin{array}{l}\text { Función } \\
\text { motora }\end{array}$ & $\begin{array}{c}\text { Sólo reflejos } \\
\text { espinales }\end{array}$ & $\begin{array}{c}\text { Sin } \\
\text { movimientos } \\
\text { intencionados }\end{array}$ & $\begin{array}{l}\text { Sin } \\
\text { movimientos } \\
\text { intencionados }\end{array}$ \\
\hline $\begin{array}{l}\text { Función } \\
\text { respiratoria }\end{array}$ & $\begin{array}{l}\text { Requiere } \\
\text { respirador }\end{array}$ & $\begin{array}{l}\text { Puede } \\
\text { requerir } \\
\text { respirador }\end{array}$ & $\begin{array}{l}\text { No requiere } \\
\text { respirador }\end{array}$ \\
\hline Pronóstico & $\begin{array}{l}\text { Estado } \\
\text { irreversible }\end{array}$ & $\begin{array}{l}\text { Puede haber } \\
\text { recuperación, } \\
\text { caer en EVP o } \\
\text { muerte cerebral }\end{array}$ & $\begin{array}{l}\text { Puede haber } \\
\text { recuperación }\end{array}$ \\
\hline
\end{tabular}

(Figura adaptada de "Muerte Cerebral" Online [Aguilar, 2013] Disponible en: http://algarabia.com/ideas/muerte-cerebral/ [Consultado el 23.07.2015]).

22 Texto original: “(...) the expectant mother has placed herself in a special class of persons who are bringing another person into existence, and upon whom that other person's life is totally dependent. Also, uniquely, the viable un-born child is literally captive within the mother's body (...)" Friedman, L. "Contemporary Cases in Women's Rights".1994, p.164. 
Otro punto importante a considerar es el fin de la existencia del concebido. Esto acontece de dos maneras: con el nacimiento, a través el cual el concebido se convierte en persona natural, y con la muerte del no-nacido, sea durante el proceso de alumbramiento- antes de que este se desvincule por completo del cuerpo de la madre- o cuando este aún se encuentra alojado en la gestante. Como consecuencia de esta última modalidad, el concebido sería también titular del derecho a una muerte digna.

Nuestro país es uno de los más conservadores de América Latina y ello es evidente al revisar nuestra legislación respecto de este tema. Otros ordenamientos, como los de Costa Rica, Chile y Argentina son bastante más abiertos a temas como "la muerte digna". No obstante, también es importante resaltar que, si bien se emplean criterios, principalmente, favorecedores en cuanto al concebido, en los últimos años, se ha incorporado determinadas bases médicas para intentar no desproteger a la gestante.

\section{A MODO DE CONCLUSIÓN}

El especial trato que recibe el concebido de forma mayoritaria en el mundo jurídico, resulta de especial importancia dado que la vida siempre debe ser prioridad entre los intereses del Estado. Un ser vivo frágil que, eventualmente, generará a una nueva per- sona natural, debe, evidentemente, captar la atención de la colectividad y ser dotado de todas las herramientas que le permitan desarrollarse de la mejor manera posible.

También es innegable que la mujer, como ser humano, es poseedora absoluta de todos los derechos que le son inherentes. La protección de su vida -en caso de encontrarse en estado de coma- y dignidad deben acompañarla hasta el fin de su existencia y, absolutamente nadie, debe ser capaz de arrebatarle esta titularidad de manera arbitraria.

En una sociedad en la que la mujer ${ }^{23}$, muchas veces, es relegada, es de suma importancia reflexionar acerca de si la protección al concebido, en determinadas circunstancias vinculadas con el tema materia del presente artículo, puede tornarse en un tipo de maltrato. Cuando nos encontramos ante situaciones límite, como las revisadas aquí, suele apreciarse una polarización bastante marcada entre médicos, legisladores y otros actores involucrados.

Es natural que el ser humano sienta la necesidad de resguardar a su especie, sobre todo en las etapas más vulnerables de su existencia. Resulta, para muchos, ser más que una obligación, un deber, y es por esto que personal de salud y magistrados, en ciertas ocasiones, han optado por apostar por el con- cebido en un afán de buscar un camino piadoso.

No obstante ello, es trascendental recordar un principio tan básico como importante: "El derecho de uno, empieza donde termina el de los demás"24, lo cual quiere decir que, si bien es correcto intentar proteger al no nacido, también será de vital importancia el respeto hacia la madre.

En otras palabras, ¿será realmente correcto tratar de salvar una vida sin tener en cuenta el contexto? La respuesta, después de revisar cifras objetivas que demuestran el decaimiento por el cual atraviesa la gestante mantenida con "vida" de manera artificial -el cual puede, incluso, afectar el desarrollo del feto- nos inclina a responder que no lo es. Lo cual demuestra, a su vez, la importancia que tiene para el Derecho desarrollarse a la par de otras ciencias.

Se deberá buscar, entonces, un punto medio elaborado sobre la base de criterios objetivos y procurando el bien mayor para poder alcanzar una solución razonable y legalmente aceptable en este tipo de casos. No todos los no-nacidos se encontrarán en la misma etapa de crecimiento y no todas las gestantes, que pierdan sus funciones vitales, reaccionarán de la misma manera al tratamiento médico.

Cada caso poseerá un distinto nivel de posibilidades de cul-

23 Es importante tener en cuenta que, en el Perú, de acuerdo con Percy Pacora Portella y Rommy Kendall Folmer (2006), en: Violencia contra la mujer en el Perú: "Tenemos una población estimada en 27 millones, de la cual el $50.1 \%$ $(13 E 628,424)$ son mujeres."

24 Frase atribuida al jurista romano Ulpiano. 
minar en un resultado positivo para el concebido. En la medida de que el cuerpo de la mujer, impedida por las circunstancias de expresar su voluntad, sea tratado con el respeto que merece, se podrá intentar salvar la existencia del hijo.

Existen tres circunstancias demarcadas que, luego de esta investigación, hemos podido identificar como generadoras de polémica en este tipo de casos. Asimismo, hemos hallado posibles respuestas objetivas a las mismas, que son las siguientes:

- Gestante fallecida con cese absoluto de actividad en todos sus sistemas internos: es plenamente identificada como un cadáver, sin discusión doctrinaria. La supervivencia del concebido dependerá totalmente de su grado de desarrollo y de lo que, médicamente, se pueda realizar por él después de extraerlo del cadáver de su progenitora.

- Gestante en estado de coma: se le deberá mantener conectada a equipos médicos si tiene posibilidades de recuperación y en la medida que esto sea positivo para el desarrollo del no-nacido mientras el trato que reciba no sea peyorativo ni merme su derecho a la dignidad.

- Gestante con muerte cerebral: legalmente, en el Perú, debe ser reconocida como una persona fallecida. La supervivencia del concebido dependerá de una serie de criterios médicos objetivos. La decisión de ser desconectada, usualmente, recae sobre los familiares correspondientes de acuerdo con la jurisprudencia internacional revisada.

\section{RefERENCIAS BIBLIOGRÁFICAS}

Aguilar, P. "Muerte Cerebral" Online (2013). Disponible en: http://algarabia.com/ ideas/muerte-cerebral/ (Consultado el 23.07.2015).

Amorim Biondo, C.; Paes da Silva, MJ; Dal Secco, LM. (2009). "Distanasia, eutanasia y ortotanasia: percepciones de los enfermeros de unidades de terapias intensiva e implicaciones en la asistencia" En: Rev Latino-am Enfermagem 2009 setembro-outubro; 17(5) (Online). Disponible en internet: http://www. scielo.br/pdf/rlae/v17n5/ es_03.pdf (Consultado el 29.09.15)

Busquets et al. "Consideraciones sobre el embrión humano" (2009). En: Bioética y debat. Volumen 15, Número 57, Monográfico 2009. (Online). Disponible en internet: http: //www.ibbioetica.org/es/img/home/ BD_57_esp_F (Consultado el 10.07.15).

Candelaria, et al. (2004). "Enciclopedia Lúmina Siglo XXI". Bogotá: Grupo Editorial Norma.

Calvo Meijide, A., " "El nasciturus como sujeto de Derecho. Concepto constitucional de persona frente al concepto pandectista-civilista". Pg. 291. (Online). Disponible en internet: http://www.
aebioetica.org/rtf/06BIOETICA54.pdf (Consultado el 10.07.15).

Cruz Ponce, L. "El nasciturus". En: Boletín Mexicano de Derecho Comparado, México, nueva serie, año XXIII, núm. 67, enero-abril, p.38, 1990

De la Fuente-Hontañón, Rosario (2011). "La protección de la vida y la dignidad de la persona humana en el derecho peruano." En: Repositorio institucional PIRHUA (Online). Universidad de Piura, Disponible en internet: http://pirhua. udep.edu.pe/bitstream / handle/123456789/1632/ Proteccion_de_la_vida_y_ dignidad_de_la_persona_ humana.pdf? sequence $=1$ (Consultado el 29.09.15)

Fernández Sessarego, C. (1988). "Tratamiento jurídico del concebido", en Libro Homenaje a Mario Alzamora Valdez. Lima: Cultural Cuzco.

Fernández Sesarego, C. (1990). Nuevas Tendencias en el Derecho de las Personas. Lima: Universidad de Lima.

Friedman, L. (1994). “Contemporary Cases in Women's Rights". Wisconsin: Univ of Wisconsin Press.

González, L. (2006). De la bioética-al bioderecho: libertad, vida y muerte. Madrid: Universidad Pontifica Comillas.

Juárez, A.; Carrillo, C.; Romero,S.; Motta, E. (1999). “Operación cesárea postmortem con superviven- 
cia fetal. Informe de un caso". En: Revista Médica del Hospital General de México S.S.. Vol. 62, Núm. 2. Abril-Junio 1999.

López, M. y Abellán, JC. (2009). Los códigos de la vida". Madrid: Homolegens.

Mejía R. et al. (2008). "Muerte cerebral en una embarazada y sobrevida del feto". ISSN 0025-7680 En: MEDICINA, Buenos Aires.
Morales Godo, J. (1997). Hacia una Concepción Jurídica Unitaria de la Muerte. Lima: PUCP.

Pacora Portella, P., Kendall Folmer, R. (2006). Violencia contra la mujer en el Perú. En: Revista Diagnóstico. Vol. $45 \mathrm{~N}^{\circ} 4$ octubre - diciembre 2006 (Online). Disponible en internet en: http: / / www. fihu-diagnostico.org.pe/revista/numeros/2006/oct-dic/173-176. html (Consultado el 04.08.15).

Sgreccia, E. (2009). Manual de Bioética I - Fundamentos y ética biomédica. Madrid: Biblioteca de Autores Cristianos.

Spota, AG. (1949). Tratado de Derecho Civil. Tomo I, Volumen 3. Buenos Aires: Editorial Depalma. 